\title{
Relation of third and fourth heart sounds to blood velocity during left ventricular filling
}

\author{
FEDERICO VANCHERI, * DEREK GIBSON
}

From the Cardiac Department, Brompton Hospital, London

SUMMARY To investigate the relation between changes in left ventricular inflow velocity and the timing of third and fourth heart sounds, simultaneous phonocardiograms and continuous wave $\vec{i}$ Doppler traces were recorded in 48 patients (aged 17-78) with heart disease and in 21 normal $\vec{A}$ children. The onset of the first vibration of the third heart sound coincided with peak left ventricular inflow blood velocity to within $5 \mathrm{~ms}$ in all but two of the patients. The mean (SD) 인 difference between the two events was 5 (5) ms, which did not differ significantly from zero. The $\vec{T}$ relation was similar in patients with primary myocardial disease (11), and in those with valve $\frac{\mathbb{D}}{0}$ disease (26), hypertension (five), and coronary artery disease (four). In the normal children, the mean interval was $2 \cdot 5$ (5) ms-not significantly different from zero. By contrast, the first deflection of the fourth heart sound consistently preceded the timing of peak atrial inflow velocity by $55 \vec{\varphi}$ (10) ms. Agreement was much closer between the onset of atrial flow and the onset of the atrial $0^{\infty}$ sound (mean difference 1 (5) ms, not significantly different from zero). Gallop sounds seem to be 0 closely related to changes in ventricular inflow velocity, and thus to the effects of forces acting on blood flow. The forces underlying the third sound seem to arise within the ventricle and are responsible for sudden deceleration of flow during rapid ventricular filling. The fourth sound, occurring at the onset of the "a" wave, is more likely to arise from dissipation of forces causing acceleration of blood flow-that is, atrial systole itself.

The third and fourth heart sounds, termed "gallop sounds" by Bouillaud in $1847^{1}$ are widely recognised to be of great clinical importance as evidence of ventricular disease. Despite this long history their exact origin remains controversial though they are generally believed to be related in some way to ventricular filling. We compared the timing of the third and fourth heart sounds with that of ventricular filling velocities as shown by continuous wave Doppler echocardiography. In this way, we hoped to explore their relation to the timing of blood flow and thus to the forces governing its rate of change.

\section{Patients and methods}

We investigated 48 patients ( 29 men, 19 women) aged 17-78 years with clinical evidence of left ventricular disease or non-rheumatic mitral regurgitation. The table gives the underlying diagnoses and occurrence Requests for reprints to Dr Derek Gibson, Cardiac Department,
Brompton Hospital, Fulham Road, London SW3 6HP.

^Present address: Corso Vittorio Emanuele 101, 93100 Caltanissetta, Sicily.

Accepted for publication 19 October 1988 of added sounds. We also studied 21 children (15 boys, six girls) aged 4-13 years. A third or fourth heart sound was audible in all the individuals studied.

Examinations by continuous wave Doppler were performed with a $2 \mathrm{MHz} 1 \mathrm{~cm}$ transducer positioned at the apex and directed towards the mitral valve. The direction of the ultrasound beam was always to the left and lateral to the characteristic velocities recorded in the left ventricular outflow tract during $D$ ejection and thus distinguished mitral from tricuspid flow. A Doptek spectral analyser was used, and the N transducer was angled, with the aid of the audio

Table Causes of added sounds

\begin{tabular}{llll}
\hline Diagnosis & III alone & IV alone & $I I I+I V$ \\
\hline Hypertrophic cardiomyopathy (6) & 1 & 3 & 2 \\
Aortic stenosis (11) & 9 & 1 & 1 \\
Hypertensive heart disease (5) & 1 & 2 & 2 \\
Aortic regurgitation (7) & 4 & 2 & 1 \\
Mitral regurgitation (8) & 5 & 0 & 3 \\
Dilated cardiomyopathy (5) & 4 & 1 & 0 \\
Ischaemic heart disease (4) & 1 & 1 & 2 \\
Restrictive cardiomyopathy (2) & 1 & 1 & 0 \\
\hline
\end{tabular}

III, third heart sound; IV, fourth heart sound. 
signal, to obtain maximum velocities of forward mitral flow, independent of mitral cusp artefacts. The delay imposed by spectral analysis was measured directly and found to be $<5 \mathrm{~ms}$. The standard lead II of the electrocardiogram and the phonocardiogram were both recorded simultaneously, the latter with a Leatham microphone from two positions: from the apex, with a low frequency filter, where the added sounds were most clearly audible; and from the left sternal edge, with a medium frequency filter, where splitting of the second sound was most obvious. Apexcardiograms were recorded with a Cambridge Instruments transducer with a time constant of $4 \mathrm{~s}$ from the apex, along with simultaneous phonocardiograms and the standard lead II of the electrocardiogram. All recordings were made with a photographic strip chart recorder at a paper speed of $100 \mathrm{~mm} / \mathrm{s}$.

The following time intervals were measured with callipers for each patient:

(a) The time interval between the peak flow velocity on the Doppler trace during early diastole and the onset of the first vibration of the third heart sound. This was either measured directly, or, in a minority when the third sound and the Doppler cardiogram could only be recorded from the same or overlapping positions on the thorax, as the difference between the intervals from the onset of the second heart sound to peak velocity of early filling and the first vibration of the third sound.

(b) The interval from the first vibration of the third sound to the " $f$ " point of the rapid filling wave of the apexcardiogram.

(c) The interval between peak flow velocity during atrial systole to the onset of the first vibration of the fourth heart sound.

(d) The interval from the onset of the flow velocity increase at the start of atrial systole to the onset of the first vibration of the fourth heart sound.

All measurements were averaged over five successive beats and given to the nearest $5 \mathrm{~ms}$. Data are expressed as mean (1 SD). The statistical significance of differences between mean values was investigated using Student's $t$ test.

\section{Results}

\section{THIRD HEART SOUND}

The first vibration of the third sound corresponded to within $5 \mathrm{~ms}$ with the peak velocity recorded on the Doppler trace in all but two of the 37 patients (figs 1 and 2). In the whole group, the mean time interval between the peak velocity and third heart sound was 5 (5) ms. There was no significant difference between values in patients with mitral regurgitation (eight cases) and those with primary left ventricular

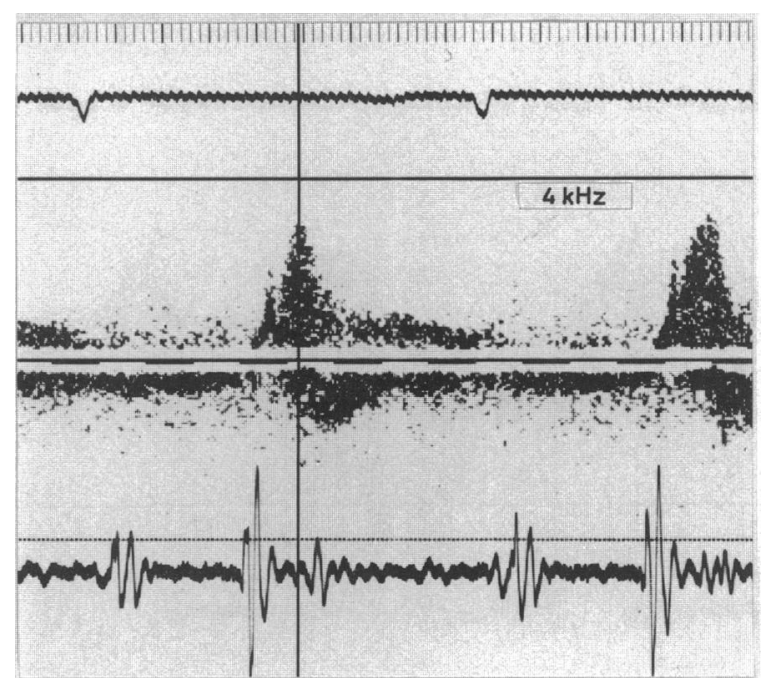

Fig 1 Simultaneous phonocardiogram, apical Doppler echocardiogram, and electrocardiogram from a patient with dilated cardiomyopathy showing a third heart sound.

Vertical line represents timing of peak left ventricular inflow velocity on the Doppler recording which corresponds with the onset of the third sound.

disease ( 29 cases). In 16 of the normal children the onset of the third sound corresponded to within $5 \mathrm{~ms}$ of the peak velocity-preceding it in two, and in three following it by $10 \mathrm{~ms}$. The mean difference between peak velocity was $2.5(5) \mathrm{ms}$. Neither of these mean values was significantly different from zero.

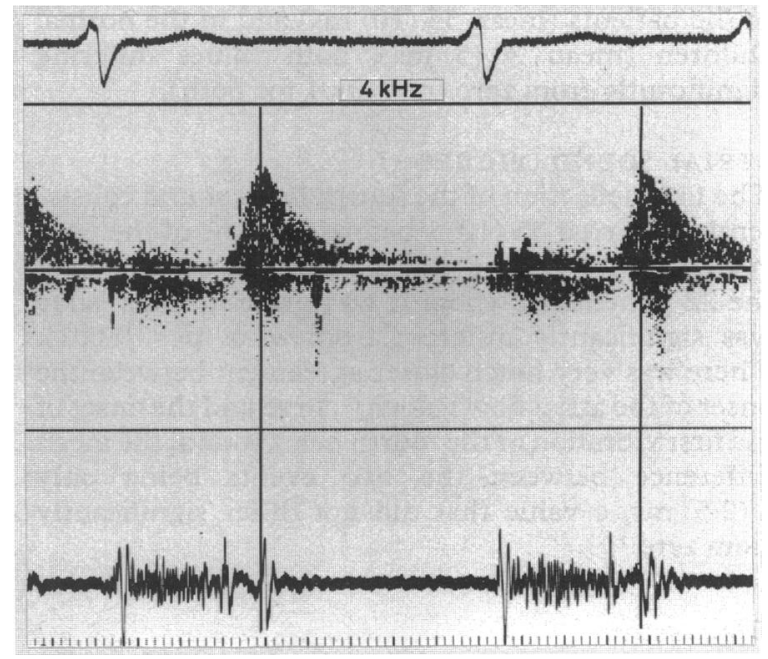

Fig 2 Simultaneous phonocardiogram, apical Doppler echocardiogram, and electrocardiogram from a patient with non-rheumatic mitral regurgitation. The vertical line shows the peak mitral flow velocity and corresponds with the initial vibration of the third heart sound. 


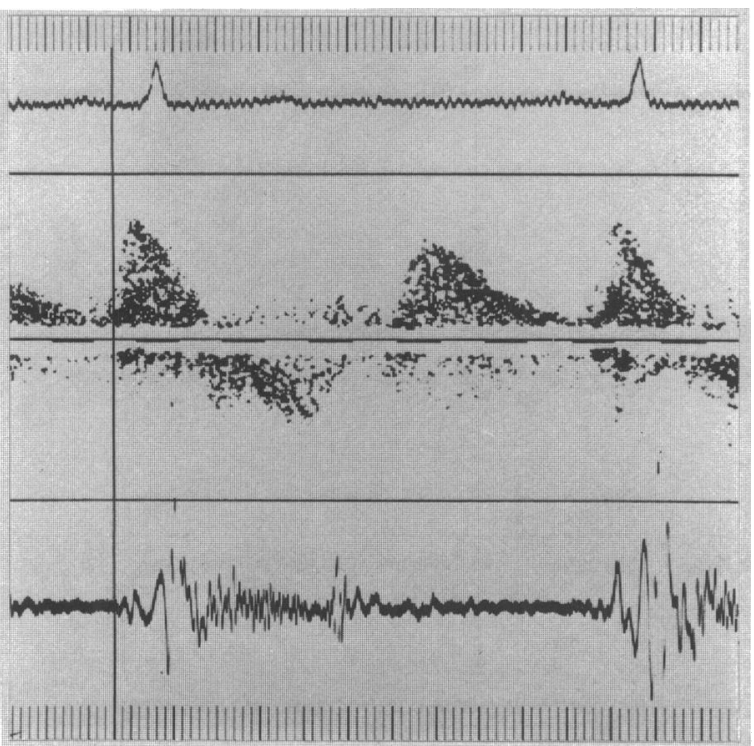

Fig 3 Simultaneous phonocardiogram, Doppler echocardiogram, and electrocardiogram from a patient with hypertensive heart disease and a fourth heart sound. Vertical line represents the onset of the fourth sound. This corresponds with the onset rather than the peak of the flow velocity trace during the " $a$ " wave. (Doppler calibration: full scale deflection $= \pm 4 \mathrm{kHz}$.)

Satisfactory apexcardiograms were obtained in 33 patients and 16 of the normal children. The first vibration of the third heart sound consistently preceded the " $f$ " point of the apexcardiogram both in the patients (mean $55(10) \mathrm{ms}$ ) and in the normal children (mean $20(5) \mathrm{ms}$ ), both values differing significantly from zero ( $p<0.01$ for both).

\section{ATRIAL SOUND (FIGURE 3)}

The first deflection of the fourth heart sound consistently occurred 55 (10) s before the time of the peak velocity of the "a" wave of the Doppler trace in all of the 22 patients in whom it was present. This value was significantly different from zero $(p<0.001)$. There was very much closer agreement between the onset of the atrial flow velocity curve and the onset of the first vibration of the fourth heart sound, the mean difference between the two events being only $1(2.5) \mathrm{ms}$, a value that did not differ significantly from zero.

\section{Discussion}

Despite their clinical importance, there is still no consensus on the genesis of the third and fourth heart sounds. It seems to be generally agreed that they are related, at least indirectly, to ventricular filling, the third heart sound to the rapid filling phase and the $c$ fourth to atrial systole. The original evidence for this $\overrightarrow{\vec{F}}$

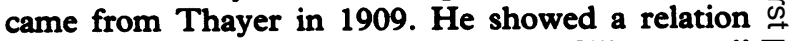
between the third sound and the "rapid filling wave" of the apexcardiogram. ${ }^{2}$ Since then it has become $\frac{\bar{D}}{\bar{D}}$ apparent that events on the apexcardiogram are not $\stackrel{5}{\vec{D}}$ closely related to volume changes in the left ventricle $\unrhd$ during diastole; in particular the " $O$ " point is independent of mitral valve opening, and the peak $\vec{P}$ rate of volume increase usually precedes the "rapid filling wave"3 although the third sound frequently coincides with the "f " point. Angiographic studies have shown that ventricular filling rates in patients with a third heart sound can be high or low." The 9 third heart sound occurred 50 (40) ms after the peak i rate of increase of the transverse dimension of the left $\vec{A}$ ventricle measured by $\mathbf{M}$ mode echocardiography and there was a suggestion of a relation between a 5 discontinuity on the dimension trace and the end of $\vec{T}$ rapid filling. ${ }^{3}$ More recently, though, experimental ${ }^{56}$ T and limited clinical ${ }^{7}$ studies have both shown an association between the third sound and sudden $\stackrel{2}{2}$ reduction in the rate of increase of the long axis but not the minor axis 48 (21) $\mathrm{ms}$ after the corresponding $\varnothing$ event on the minor axis. This delay is thus very. similar to the interval previously reported by Prewitt et $\mathrm{al}^{3}$ between the third sound and the peak rate of increase in minor axis. The mechanism underlying this sudden deceleration has not been established, though the persistence of a third heart sound after $\stackrel{\mathbb{Q}}{2}$ replacement of the mitral and tricuspid valves $\overrightarrow{\vec{O}}$ showed that the subvalve apparatus was not neces- $\frac{3}{3}$ sarily involved. ${ }^{89}$

The timing of the third heart sound is also closely? related to changes in atrial and ventricular pressure. During the early period of ventricular filling, ventricular pressure first falls, and then rises rapidly, causing the atrioventricular pressure gradient to reverse, leading to a deceleration of ventricular inflow. It is during this period of deceleration that the $\frac{\circ}{3}$ third sound occurs. ${ }^{10} 11$ Our results thus closely agree $\circ$ with these findings. The third heart sound occurred at the time of peak velocity of inflow, marking the time at which acceleration had ceased. Acceleration of fluid is associated with a pressure gradient in the $\sigma$ same direction as flow, and deceleration with a $N$ gradient in the reverse direction. Our finding that the $\underset{\mathrm{N}}{\mathrm{N}}$ third sound occurred when acceleration had ceased is $\sigma$ thus equivalent to the results of van de Werf et al who showed that it followed reversal of the pressure gradient. ${ }^{1011}$ The sudden change from acceleration to $\stackrel{\infty}{\rightarrow}$ deceleration requires the operation of a force and the $\square$ dissipation of energy; it is thus likely that this energy $\overrightarrow{0}$ appears, in part, as the mechanical vibrations which $\stackrel{\odot}{\Phi}$ form the third heart sound.

Like the third heart sound, the fourth sound is 
associated with a period of increased rate of ventricular inflow. Its audibility is evidence of ventricular disease, ${ }^{1213}$ although a low frequency event can sometimes be recorded phonocardiographically in normal subjects during atrial systole. ${ }^{14}$ As in early diastole, its timing has been related to reversal of the atrioventricular pressure gradient with atrial systole. ${ }^{1011}$ Unlike the third sound, however, we found that the onset of the fourth heart sound corresponded with the onset rather than with the peak of the flow velocity trace. The force generating the fourth heart sound, therefore, seems to be the one that accelerates the blood into the ventricle, and so is the result of left atrial systole rather than some ventricular factor impeding blood flow as occurs with the third sound.

Doppler estimates of blood flow velocity are subject to certain technical limitations. They are confined to the region examined by the ultrasound beam, and so may not necessarily be representative of the entire ventricular inflow. However, the change in velocity with time is so abrupt that it seems unlikely that there could be wide differences within the column of blood moving into the ventricle without pronounced velocity gradients developing, and there is no experimental evidence of this. The use of continuous wave rather than pulsed Doppler echocardiography has the advantage that the maximum blood velocity is measured, regardless of its distance from the transducer; there seemed no particular reason to believe that the timing of added sounds would be related to blood velocities at specific positions within the ventricle such as the region just below the mitral cusps. The patients had clear evidence of left sided disease, so their third heart sounds probably arose from the left ventricle. A right sided third heart sound would not necessarily be expected to bear any consistent relation to the timing of left sided filling. An example is given in fig 4, from a patient with right ventricular disease, where the timing of the third sound was closely related to inflow velocities in the superior vena cava; peak mitral flow occurred 50 ms later.

The characteristic relation between ventricular filling velocities and the timing of added sounds provides information about the genesis of these sounds, but does not explain why they are audible in some patients and not in others. Presumably, ventricular diastolic properties must also be taken into account in predicting the nature of the forces that modify blood flow, in determining their timing, and in governing the extent to which the resulting energy dissipation will be converted to longitudinal vibrations audible as sound. For the fourth heart sound, atrial systolic function as well as ventricular diastolic properties must also be considered. Nevertheless, our results extend traditional lines of thought about

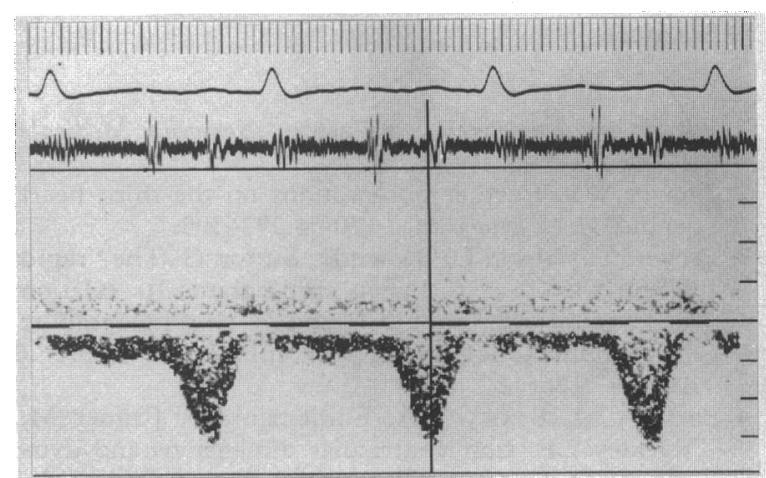

Fig 4 Simultaneous phonocardiogram, electrocardiogram, and Doppler echocardiogram from a patient with right ventricular disease caused by anterior mediastinal radiotherapy. The diagnosis of restriction was confirmed at thoracotomy. The Doppler echocardiogram is recorded from the suprasternal notch and shows the flow velocity in the superior vena cava. The vertical line shows the peak blood velocity which corresponds with the onset of a third heart sound. (Doppler calibration: full scale deflection $= \pm 8$ kHz.)

the genesis of these abnormal sounds. They confirm their close relation to the processes underlying ventricular filling. ${ }^{15}$

Our results indicate that the physiological third sound has a similar time relation to ventricular inflow velocities and their rate of change as that associated with mitral regurgitation or ventricular disease. We did not confirm the recent suggestion that the third sound is caused by the ventricle impinging on the thoracic cage. ${ }^{16}$ Our observations raise the possibility that the Doppler trace may be a useful reference for analysing these events, particularly when their underlying mechanism is not clear-in some cases, for example, a summation gallop may result primarily from atrial systole, when the onset of the sound coincides with that of the increase in flow velocity, while in others it has the characteristics of a third sound even though it occurs after the $P$ wave of the succeeding beat.

Finally, our results allow a unitary concept to be developed to explain the genesis of these added sounds - that is, they are the result of the dissipation of energy generated by the forces that cause changes in blood flow velocity. If this is proved, it gives a basis whereby these sounds, whose clinical importance has been so long and widely recognised, can be related more closely to the fundamental processes of ventricular filling, and thus to overall diastolic function in health and disease. 


\section{References}

1 Potain C. Les bruits de galop. Semaine Medicale 1900;20:175-6.

2 Thayer WS. Further observations on the third heart sound. Arch Intern Med 1909;4:297-305.

3 Prewitt T, Gibson D, Brown D, Sutton G. The "rapid filling wave" of the apex cardiogram. Its relation to echocardiographic and cineangiographic measurements of ventricular filling. Br Heart $J$ 1975;37:1256-62.

4 Porter CM, Baxley WA, Eddleman EE, Frimer M, Rackley CE. Left ventricular dimensions and dynamics of filling in patients with gallop heart sounds. 27Am J Med 1971;50:721-7.

5 Ozawa Y, Smith D, Craige E. Origin of the third heart sound; I. Studies in dogs. Circulation 1983;67:393-8.

6 Ishimitsu T, Smith D, Berko B, Craige E. The origin of the third heart sound: comparison of ventricular wall dynamics in hyperdynamic and hypodynamic types. J Am Coll Cardiol 1985;5:268-72.

7 Ozawa Y, Smith D, Craige E. Origins of the third heart sound: II. Studies on human subjects. Circulation 1983;67:399-404.
8 Marshall JC, Gibson DG. Origin of the third heart sound [Letter]. $\mathrm{Br}$ Med J 1969;iii:778.

9 Gianelly RE, Popp RL, Hultgren HN. Heart sounds in patients with homograft replacement of the mitral valve. Circulation 1970;42:309-21.

10 van de Werf F, Minten J, Carmeliet P, de Geest H, Kesteloot $\mathrm{H}$. The genesis of the third and fourth heart sounds. A pressure-flow study in dogs. J Clin Invest 1984;73:1400-7.

11 van de Werf F, Boel A, Geboers J, et al. Diastolic $\vec{\circ}$ properties of the left ventricle in normal adults and in patients with third heart sounds. Circulation 1984;69:1070-8.

12 Potain C. Du bruit de galop. Gazette des Hôpitaux 1880;53:529-31.

13 Weitzman $\mathrm{D}$. The mechanism and significance of the auricular sound. Br Heart J 1955;17:70-8.

14 Benchimol A, Desser KB. The fourth heart sound in $\vec{f}$ patients without demonstrable heart disease. $A m$ 의 Heart J 1977;93:297-301.

15 Crevasse L, Wheat MW, Wilson JR, Leeds RF, Taylor $\vec{T}$ $\mathrm{J}$. The mechanism of the generation of the third and $\mathbb{O}$ fourth heart sounds. Circulation 1962;25:635-42.

16 Reddy PS. The third heart sound. Int J Cardiol 1985;7:213-21. 